\title{
THE ROMANIAN POPULATION'S ACCESS TO EDUCATION DURING 1990-2010
}

\author{
Maria Livia ȘTEFĂNESCU1
}

\begin{abstract}
In the present article, we will analyze the thematics QAFE, which means the Romanian population's access to the desired forms of education during 1990-2010. The data used in the current study represent the answers of the Romanians to the question QAFE present in 13 representative samples at national level (the database DQLR-RIQL). Concretely, we will use categorial data of ordinal type. We intend to create a classification of the years from the period 1990-2010, from the point of view of the QAFE problematics. In this way, in a first approach we have used the partial relation " $\leq_{o s}$ " of stochastic order which applies to ordinal categorial variables. We have extended the analysis on the thematics QAFE by proposing the indicator PSA of "progress" in order to create a bierarchy of the years from the period 1990-2010 with regard to Romanians' perception on the aforementionned aspect. The present study shows a permanent change of Romanians'opinions regarding their access to the current forms of education. Among other causes, this aspect is generated by the lack of coherence in the promotion in the educational system from Romania of educational policies that are stable and efficient in the long term.
\end{abstract}

Key words: access to education; classification; stochastic order relation; progress indicator.

\section{Introduction}

For a harmonious development on the long term of a nation we must take into account several priorities. In this context, we remark among others: the adaptation to the new demands of the current time and the development of the educational system, the efficiency of the public health system and the extension of researches in the medical field, the development of the communications system and the creation of an infrastructure which is adequate to the third milenium, the knowledge and constructive approach of the social system.

1 Researcher, The Research Institute for Quality of Life, Romanian Academy, E-mail: livia.stefanescu@gmail.com 
In this presentation we will make a first analysis of the QAFE thematics based on the effective access of the Romanian population to different forms of education. The current research takes under consideration the direct answers of the Romanians during 1990-2010 to the question QAFE: " How do you estimate the access to the educational forms desired by yourself and by your close ones?". The respondents have to choose between five possible answer variants:"very low" (code R1), "low" (code R2), "so and so" (in 2003, 2006, 2010 it was chosen the form "satisfactory", code R3), "high" (code R4); "very high" (code R5).

Within several future articles we intend to approach the following aspects:

- A hierarchization of the years 1990-2010 regarding the answers to the question QAFE as were received by the population.

- Based on the Romanians opinions, the elaboration of a prognosis model for the evolution of the real degree of accessibility of the population to the current forms of education.

- Which are the major differences between the rural and urban areas regarding the degree of accessibility to the current forms of education.

- The opinion of families with children regarding the degree of accessibility of their children to the current forms of desired education.

- The influence of the population's poverty level in the concrete selection procedure by the young people of different forms of vocational development.

- The Romanians'opinions regarding the current educational system and its comparison to other national systems from Romania (the health system, the pensions system).

- Suggesting new approaches in the educational system. Social policies which need to be promoted for the improvement of the population's acces to different forms of education.

In this presentation, we will be treating partially only the first previously signaled theme, the other themes will be the subject of future papers.

\section{Data presentation}

The current study uses the answers of the population from Romania to the question QAFE, question that was mentionned in the previous section. The statistical analysis is based on 13 samples that are nationally representative. The samples have been designed at different steps of the period 1990-2010 at the Research Institute for Quality of LifeRomanian Academy (the data bases DQLR-RIQL). In Table 1, there are mentionned the years in which data was collected and also the volumes of the respective samples. 
94 The Romanian population's access to education during 1990-2010

Table 1. The volumes of the samples from the period 1990-2010 and the percentages of the situations "not the case" (NC).

\begin{tabular}{|l|c|c|c|c|c|c|c|}
\hline Year & $\mathbf{1 9 9 0}$ & $\mathbf{1 9 9 1}$ & $\mathbf{1 9 9 2}$ & $\mathbf{1 9 9 3}$ & $\mathbf{1 9 9 4}$ & $\mathbf{1 9 9 5}$ & $\mathbf{1 9 9 6}$ \\
\hline Size & 2033 & 1502 & 1516 & 1491 & 1500 & 1509 & 1510 \\
\hline Percent NC & 22.53 & 27.50 & 16.75 & 14.08 & 7.00 & 4.71 & 6.42 \\
\hline Year & $\mathbf{1 9 9 7}$ & $\mathbf{1 9 9 8}$ & $\mathbf{1 9 9 9}$ & $\mathbf{2 0 0 3}$ & $\mathbf{2 0 0 6}$ & $\mathbf{2 0 1 0}$ & \\
\hline Size & 1218 & 1136 & 1195 & 1018 & 1133 & 1161 & \\
\hline Percent NC & 9.61 & 8.63 & 5.36 & 8.25 & 4.06 & 0.69 & \\
\hline
\end{tabular}

Source: Analysis of the database DQLR-RIQL, period 1990-2010.

The number of the situations "not the case" $(\mathrm{NC})$ is sometimes incredibly high $(27.5 \%$ in the year 1991), although the concrete formulation of question QAFE allowed the respondent to give a clear answer in the situation in which he did not have a family or children. The results of the statistical analysis regarding the $Q A F E$ problematics will certainly be more unfavorable if we will interpret the presence of the NC answer option as a negative impact on the possibility of respondents to have access to education. The incidence of NC situations is reduced drastically in the year 2010 when the number of $\mathrm{NC}$ cases represent only $0.7 \%$ from the total answers.

In the statistical models which will be promoted in the following we will neglect the options NC of the respondents. Table 2 presents an overview regarding the yearly frequencies of the effective codes R1-R5 of answers to the question QAFE.

Table 2. Frequencies of the scores R1-R5 to the question QAFE (total sample)

\begin{tabular}{|c|c|c|c|c|c|c|}
\hline Year & R1 & R2 & $\mathbf{R 3}$ & $\mathbf{R} 4$ & $\mathbf{R 5}$ & Total \\
\hline 1990 & 153 & 389 & 610 & 371 & 52 & 1575 \\
\hline 1991 & 97 & 314 & 455 & 189 & 34 & 1089 \\
\hline 1992 & 91 & 285 & 486 & 358 & 42 & 1262 \\
\hline 1993 & 111 & 321 & 395 & 412 & 42 & 1281 \\
\hline 1994 & 41 & 315 & 538 & 462 & 39 & 1395 \\
\hline 1995 & 80 & 316 & 547 & 465 & 30 & 1438 \\
\hline 1996 & 127 & 367 & 534 & 352 & 33 & 1413 \\
\hline 1997 & 99 & 280 & 403 & 291 & 28 & 1101 \\
\hline 1998 & 56 & 259 & 383 & 317 & 23 & 1038 \\
\hline 1999 & 79 & 390 & 442 & 200 & 20 & 1131 \\
\hline 2003 & 54 & 204 & 332 & 309 & 35 & 934 \\
\hline 2006 & 84 & 245 & 413 & 306 & 39 & 1087 \\
\hline 2010 & 60 & 271 & 477 & 308 & 37 & 1153 \\
\hline
\end{tabular}

Source: Analysis of the databases DQLR-RIQL, period 1990-2010. 
It is difficult that only by the visualisation of the sequence of numeric values from Table 2 we will have a correct image on the years in which the Romanian population is more satisfied by the access to education. Moreover, by the direct and comparative evaluation of the values from Table 2 we cannot easily identify the eventual steps of continuity of opinions of the population in the problematics $Q A F E$, steps that will comprise at least four consecutive years.

In a first analysis of data from Table 2, we observe that regarding the access to education the frequencies of answers "very low" (code R1) are always at least two or three times higher than the frequencies of the opinions "very high"(the answer code R5). This aspect shows a non-negligible dose of pessimism of the population in the estimation of individuals' access to education.

Moreover, from all the possibilities R1-R5 to answer the question $Q A F E$ predominates in Table 2, several times significantly, the answer code R3. The code R3 expresses a relatively uncertain opinion ("so and so"). Although sensibly different, the case of the year 1993 does not contradict the affirmation.

Taking into account the situation presented earlier it is advisable to do a data analysis for Table 2 by neglecting systematically the answer code R3 ("so and so"). This fact will determine an important information loss,extremely useful data to obtain a high accuracy in order to separate the years of the period 1990-2010.

We suggest another approach of the situation "so and so". Concretely, the interpretation of the population's answers as realisations of a categorial ordinal type variable will solve the negative aspects signaled earlier (Agresti, 2010; Azen, 2011; Cliff and Keats, 2003). So, in the estimation of the QAFE problematics the answers with the code R2 ("low") are more critical than the opinions R3 ("so and so"). Similarly, a "high" access to education (code R4) is obviously preferable to the variant "so and so" (code R3). With such an interpretation approach of the answers of the individuals to question $Q A F E$ we are no longer forced to renounce at the opinions of the persons who have opted for the variant $\mathrm{R} 3$ of answer.

In Figure 3, there are visualised yearly the distributions of the answers R1-R5 from Table 2. We remark the different behaviour of the years 1992-1994 or 1999 and the phase 2003-2010 of "relative stability" of the population's opinions. The direct interpretation of Figure 3 shows a frequent change in time of the Romanians'opinions regarding the thematics $Q A F E$.

The image from Figure 3 does not lead us to a clear classiffication of the years of the period 1990-2010 by the favourable or unfavourable apreciation by the population of the possibility of the individual to beneficiate from education.

Taking into account all the signaled aspects, in order to increase the accuracy of the data interpretation from Table 2 we will propose in the following sections several statistical models specific to categorial ordinal type variables. As references we mention in this context the following papers: Agresti, 2010; Azen, 2011; Cliff and Keats, 2003; Szekli, 1995; Sutradhar, 2014 or Stefanescu, 2015. 
Figure 3. The distributions of the population's answers to the question QAFE (period 1990-2010).

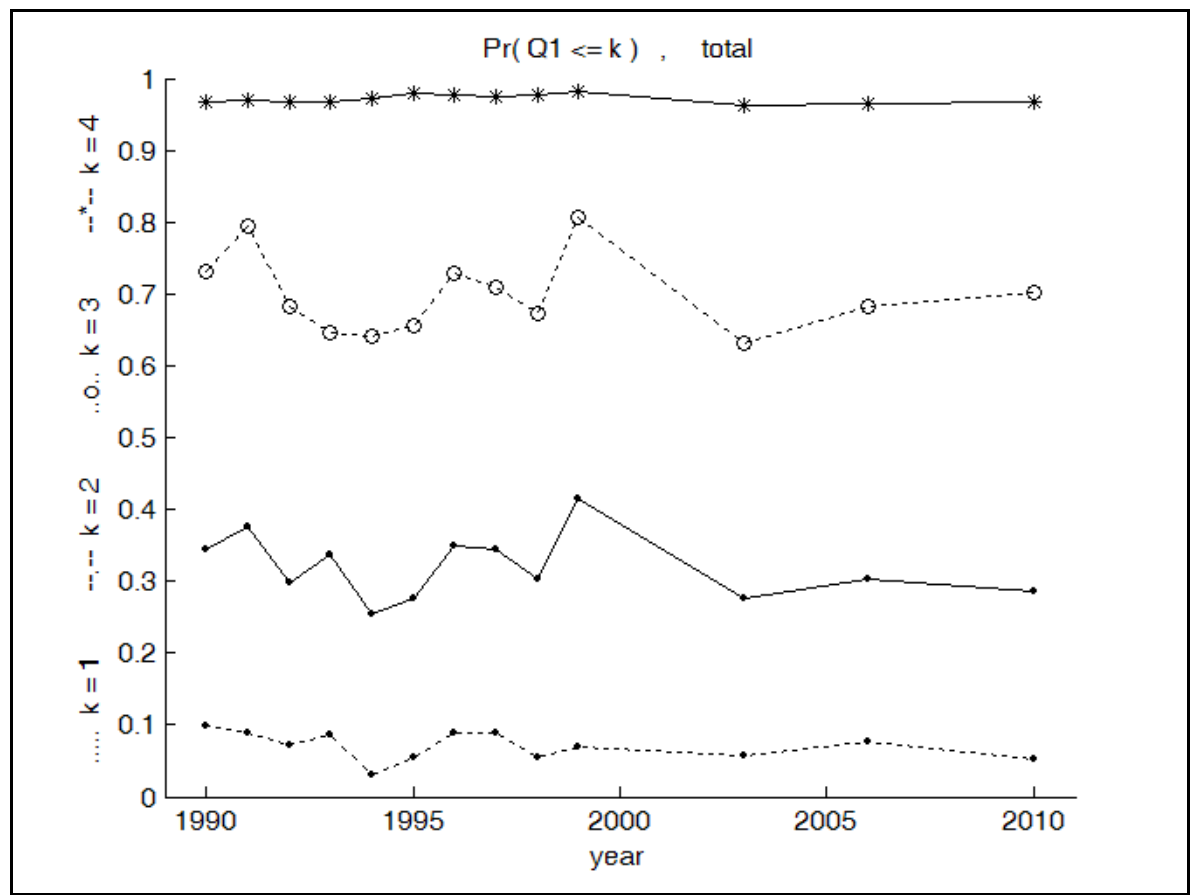

Source: Analysis of the database DQLR-RIQL, period 1990-2010

\section{Stochastic order}

Normally, in order to compare the years of the period 1990-2010 we will use a domination relation (Zheng 2007). We will evidentiate the relations between any two years by considering as a separation criterion the probabilities of the scores R1-R 5 for answering question $Q A F E$. In such a context, we choose the partial classic relation " $<_{o s}$ " of stochastic order between the distributions of two categorial ordinal type variables (Szekli, 1995). We mention the fact that in the case of random ordinal variables we can construct several stochastic dominance relations for measuring inequalities between variables (Le Breton and Peluso, 2009; Zheng, 2007).

By using the stochastic order relation " $<_{o s}$ " we will compare the $Q A F E$ distributions from Figure 3 attached to the 13 years selected from the period 1990-2010. In Table 4, there are listed the 21 stochastic dominance relations " $<$ os" which have been pointed out between the 13 years analysed. We mention the fact that in the comparisons between the probabilities that define the QAFE distributions, the error eos that is admitted in order to establish the stochastic order is null (eos $=0)$. 
Table 4. The relation " $<_{\text {os }}$ " present between the years of the period 1990-2010 $($ eos $=0)$.

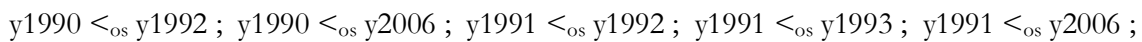
y1991 < os y2010; y1995< < os y1994; y1996< < os y1992; y1996< < os y1993; y1996< < os y1994;

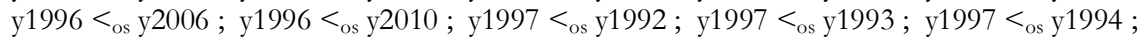

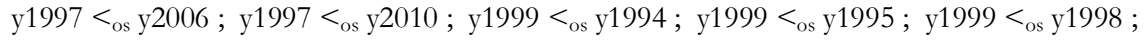
y1999< < $\mathrm{y} 2010$;

Source: Analysis of the database DQLR-RIQL, period 1990-2010

In this case, for most pairs $(y 1, y 2)$ of two years $y 1$ and $y 2$,none of the dominance relations is validated $\mathrm{y} 1<_{\mathrm{os}} \mathrm{y} 2, \mathrm{y} 1>_{\mathrm{os}} \mathrm{y} 2$ or $\mathrm{y} 1=\mathrm{y} 2$. Concretely, $67.5 \%$ of all pairs $(\mathrm{y} 1$, y2) have the years $y 1$ and $y 2$ incompatible from the point of view of the stochastic order relation " $<_{\mathrm{os}}$ " based on the QAFE criterion.

In Figure 5 are visualized the dominance relations " $<$ os" which have already been mentioned in Table 4. The relation $\mathrm{y} 1<_{\mathrm{os}} \mathrm{y} 2$ is graphically represented in Figure 5 by y1 $\rightarrow \mathrm{y} 2$.

Comparing with Table 4, Figure 5 gives us a more clear panoramic view on the population's access to education in the period 1990-2010.

Figure 5. The relations between the years of the period 1990-2010 based on the stochastic order "<os" $($ eos $=0)$.

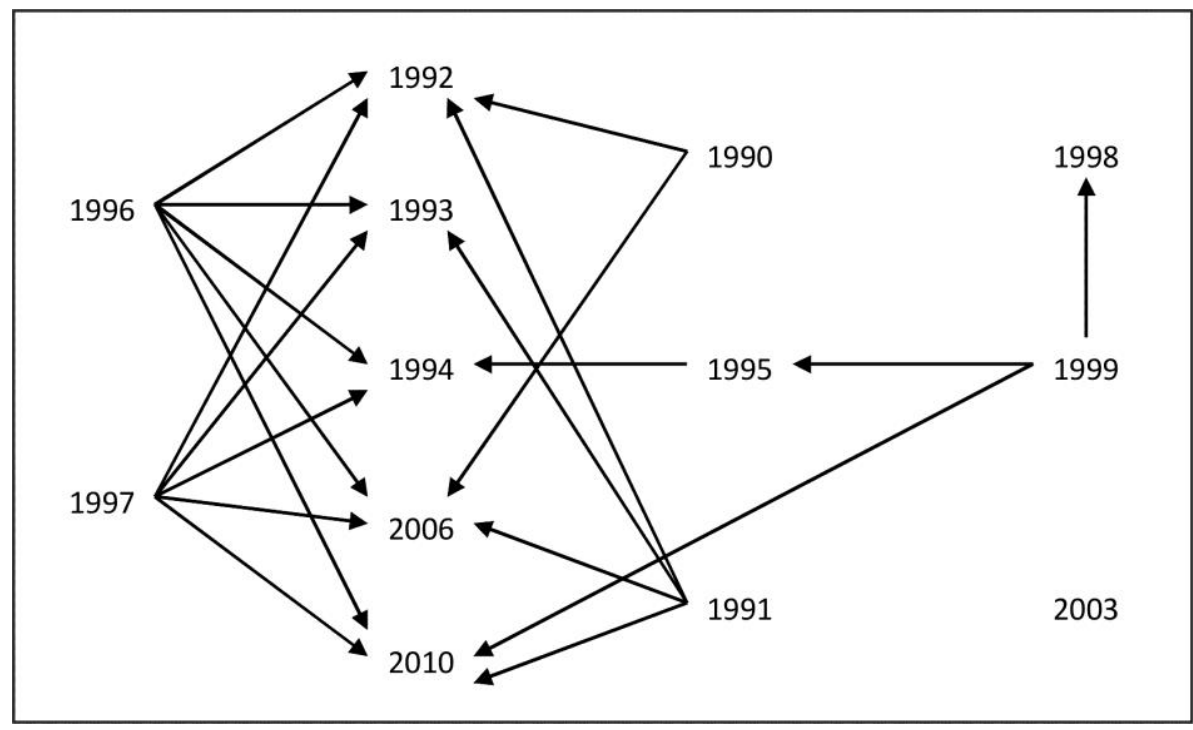

Source: Analysis of the database DQLR-RIQL, period 1990-2010 
By interpreting the graphical image from Figure 5, we remark the following aspects:

- Compared with the other years, in the phases 1992-1994 and 2006-2010, the population is relatively more content with the access to education;

- Between the group of years 1992-1994 or between the years 2006 and 2010 there is no relation of dominance, fact that can signify the lack of a clear direction in the evolution of opinions of Romanians regarding the efficiency of the QAFE thematics (Figure 5);

- The year 2003 is an atypical year and it is impossible to be compared from the point of view of the order relation " $<_{\text {os }}$ " with any of the other years of the period 19902010 ;

- The absence from Figure 5 of a chain that would comprise at least four consecutive years denotes a lack of "continuity" of the population's opinions in the QAFE problematics.

This aspect can be due to the promotion during 1990-2010 of several contradictory educational decisions which turn out to be inefficient in the long run. Such decisions have afterwards been anulled by the next government that has taken power.

\section{Progress index}

The stochastic order relation y1 $<_{o s}$ y 2 can be used to confirm the presence of a "progress" tendency from year y1 to year y2 in relation with a certain criterion. Concretely, the existence within the $Q A F E$ thematic of the inequality y $1<_{\text {os }} \mathrm{y} 2$ signifies that the population is more "content" in the year y2 compared with year y1 if we are talking about the $Q A F E$ problematics which characterizes the distributions of the categorial ordinal variables y1 and y2.

The stochastic order relation " $<_{\text {os }}$ "which is applied to a pair of distributions presents the disadvantage that we cannot establish all the time a dominance relation between the two distributions composing the pair. We mention the case of the years 1990 and 1999 (Figure 5). We mention the presence in Figure 5 of a large number of pairs of years which are not comparable " $<_{\mathrm{os}}$ " $(67.5 \%)$. Also in Figure 5 we remark the year 2003 which cannot be compared with any other year when we use the relation " $<_{\text {os }}$ ". Nevertheless, if we interpret the raw data from Table 2 for the years 1999 and 2003 or the graphical image from Figure 3 of the distributions of those two years, we deduce that in the year 2003 Romanians are more content by the access they have to education. We mention the fact that by the relation " $y 1<_{\mathrm{os}} \mathrm{y} 2$ " it is not stated the size of the gap of "progress" between the years y2 and y1.

Under these conditions we need to define an indicator PRO of "progress" that solves all the problems previously mentioned. The PRO index must mandatorily verify a set of 10 conditions (Stefanescu 2015).Moreover, the PRO coefficient must be compatible with the relation of stochastic order " $<_{\mathrm{os}}$ ". Concretely, if $\mathrm{y} 1 \leq_{\mathrm{os}} \mathrm{y} 2$ then we necessarily have $\operatorname{PRO}(y 1) \leq \operatorname{PRO}(y 2)$. We mention the fact that the PSA index which was proposed by Stefanescu in 2015 is of type PRO. 
We remind several important properties of the PSA coefficient (Stefanescu 2015). The PSA indicator varies in the interval $[0,1]$. The smallest value of the PSA index (PSA = 0 ) is obtained if the entire population has selected the variant of answer R1 ("very low") to the question QAFE. We have the maximum value of the PSA coefficeint (PSA = 1) when the total population estimates as being "very high"( code R5) the access to the desired forms of education. In Table 6, there are listed the values of the index PSA of "progress" in the case of the $Q A F E$ problematics.

Table 6. The values at the QAFE problematics for index PSA for the period 1990-2010

\begin{tabular}{|l|c|c|c|c|c|c|c|}
\hline Year & $\mathbf{1 9 9 0}$ & $\mathbf{1 9 9 1}$ & $\mathbf{1 9 9 2}$ & $\mathbf{1 9 9 3}$ & $\mathbf{1 9 9 4}$ & $\mathbf{1 9 9 5}$ & $\mathbf{1 9 9 6}$ \\
\hline PSA & 0.4650 & 0.4427 & 0.4950 & 0.4908 & 0.5256 & 0.5085 & 0.4641 \\
\hline Year & $\mathbf{1 9 9 7}$ & $\mathbf{1 9 9 8}$ & $\mathbf{1 9 9 9}$ & $\mathbf{2 0 0 3}$ & $\mathbf{2 0 0 6}$ & $\mathbf{2 0 1 0}$ & \\
\hline PSA & 0.4703 & 0.4981 & 0.4319 & 0.5179 & 0.4933 & 0.4980 & \\
\hline
\end{tabular}

Source: Analysis of the database DQLR-RIQL, period 1990-2010

The PSA values listed in Table 6 are relatively very small, below the threshold of 0.53 . We mention that an efficient solution to the problematics of population access to the current forms of education supposes PSA values that are above the threshold of 0.7.

By ordering decreasingly the values of the PSA index we create a hierarchization of the analyzed years from the period 1990-2010 (Table 7).

Table 7. Hierarchization of the years of the period 1990-2010 regarding the decreasing ordering of PSA values for the QAFE thematic.

\begin{tabular}{|l|c|c|c|c|c|c|c|}
\hline Rank & $\mathbf{1}$ & $\mathbf{2}$ & $\mathbf{3}$ & $\mathbf{4}$ & $\mathbf{5}$ & $\mathbf{6}$ & $\mathbf{7}$ \\
\hline Year & 1994 & 2003 & 1995 & 1998 & 2010 & 1992 & 2006 \\
\hline Rank & $\mathbf{8}$ & $\mathbf{9}$ & $\mathbf{1 0}$ & $\mathbf{1 1}$ & $\mathbf{1 2}$ & $\mathbf{1 3}$ & \\
\hline Year & 1993 & 1997 & 1990 & 1996 & 1991 & 1999 & \\
\hline
\end{tabular}

Source: Analysis of the database DQLR-RIQL, period 1990-2010

Although atypical in Figure 5, the year 2003 proves to be an "optimistic" year from the point of view of the approach of the QAFE problematics. Therefore, the year 2003 occupies the second position in the classification from Table 7 based on the PSA indicator.

The population's opinion regarding the access of individuals to forms of education is most favourable in the year 1994. Romanians have in the year 1999 the worst opinion on the QAFE problematics (Table 7). 
Figure 8. Fluctuation in time of the PSA index of "progress"

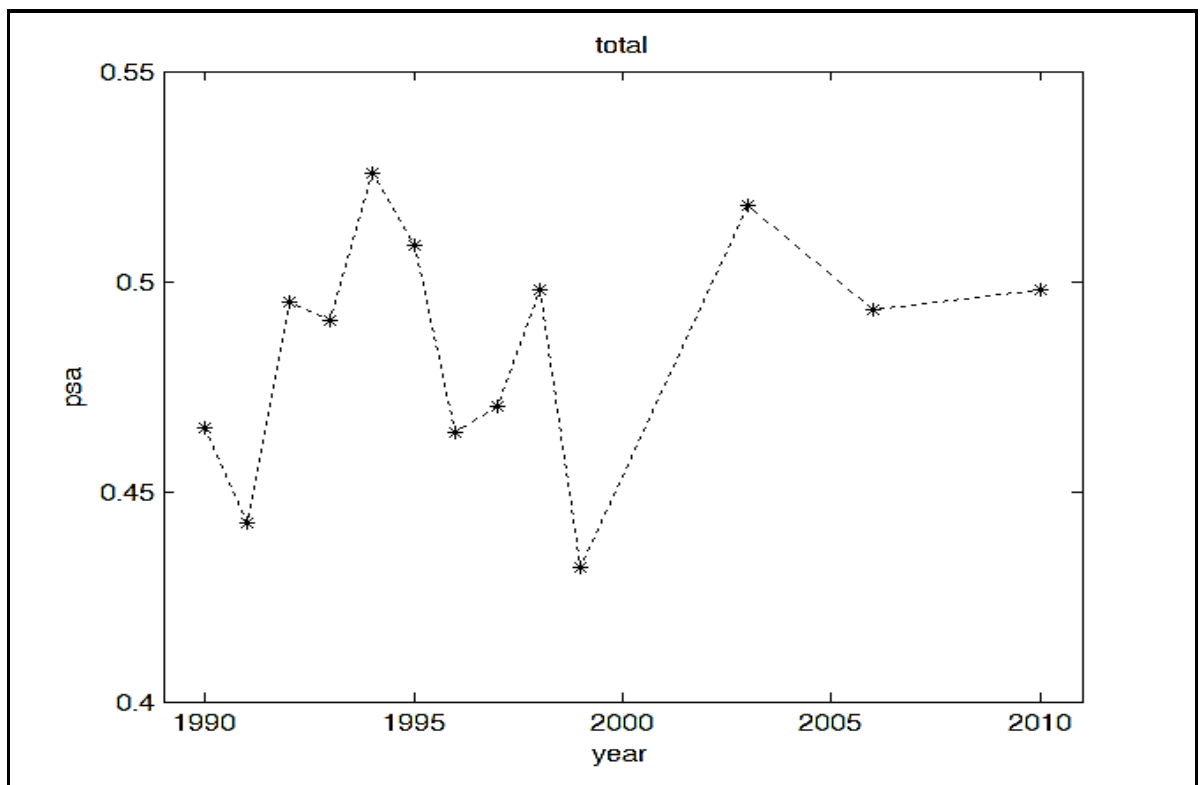

Source: Analysis of the database DQLR-RIQL, period 1990-2010

The values of the PSA index of "progress" vary in the period 1990-2010 between 0.432 and 0.526 (Table 6). A clearer image of the fluctuation in time of the PSA coefficient is presented in Figure 8.

The variation of the PSA indicator is relatively consistent and does not suppose a tendency (Figure 8). Moreover, we cannot identify in Figure 8 a step that would comprise at least four consecutive years in which we will keep an increasing tendency of the PSA values.

The aspects mentioned in the graphics from Figure 8 denote a lack of "continuity" in the population's opinions regarding the QAFE thematics. This could be due to the absence of a long-term vision of possible negative effects generated by the promoted educational policies. We also remark frequent modifications and sometimes contradictions of the ministerial decisions adopted in the period 1990-2010.

Nevertheless, in the steps 1991-1994 and 1999-2003 we remark tendencies of improving the Romanians 'perception in the QAFE problematics (Figure 8).

\section{Conclusions}

In order to bring some clarifications regarding the population's access to forms of desired education we have preferred a direct analysis of Romanians' answers to the 
question QAFE: "How do you estimate the access to the forms of education desired by yourself and by your close ones?". The $Q A F E$ problematics is present in 13 nationally representative samples from the period 1990-2010 (the database DCV-ICCV).

For the statistical analysis of the QAFE thematics from the period 1990-2010 was applied a unitary work methodology based on the classical stochastic relation " $\leq_{\mathrm{OS}}$ " for ordering the distributions of ordinal type categorial variables. In the same time it has been proposed the PSA indicator of "progress" which is compatible with the stochastic order relation " $\leq_{\mathrm{os}}$ ".

Figure 5 shows the relations between the years of the period 1990-2010 by using the inequality relation " $\leq_{\mathrm{os}}$ ". Therefore we obtain a structural characterization of the period 1990-2010 from the point of view of Romanians'access to their desired forms of education (Figure 5).

Table 6 presents the evolution of longitudinal values of the PSA index of "progress" with relation to the $Q A F E$ problematics.All these values are relatively small and they do not surpass the threshold of 0.53. This fact attests to a partial and inefficient solution in the long term of the QAFE problem.

A hierarchization of the years from the studied period is given in Table 7 . In the year 1994, the Romanians'opinions are most favourable to the QAFE thematics. At a difference of only five years, in the year 1999 Romanians are discontent in the highest degree by the access to forms of education.

Figure 8 presents the longitudinal fluctuations of the values of the PSA coefficient which shows the level of satisfaction of the respondents to the question QAFE. We remark a relatively consistent fluctuation of the PSA index in the interval $[0.432,0.526]$. At the same time, we do not identify in Figure 8 a general increasing or decreasing tendency of the PSA values. Moreover, we cannot select from the period 1990-2010 a single step that comprises at least four consecutive years and in which we can observe clearly a certain evolutionary tendency of the population's opinions (Figure 8). This fact proves the lack of "continuity" of the population's opinions regarding the QAFE thematics.

In conclusion, a significant part of the population is disappointed by the possibility to effectively beneficiate from the educational forms desired. The permanent change, from year to year, of the opinions of the questionned individuals in the $Q A F E$ problematics is generated by the absence of a unitary vision at government level, on the long term, for the promotion of certain types of educational policies.

The partial results already obtained but not included in the present paper show for the QAFE problematics large differences between rural and urban areas, the paramount importance that have the individual poverty level, the discrimination of families with a lot of children, the strong link between the eficiency of the present educational system and the punctual aspect of QAFE. The policy proposals destined to improve the current situation will have to keep in mind the mentionned aspects. 
102 The Romanian population's access to education during 1990-2010

\section{References}

Agresti, A. (2010). Analysis of ordinal categorical data, New Jersey: John Wiley and Sons, second edition.

Azen, R. (2011). Categorical data analysis for the behavioral and social sciences, New York: Routledge, Taylor \& Francis Group.

Le Breton, M., Peluso, E. (2009) Third-degree stochastic dominance and inequality measurement, The Journal of Economic Inequality 7, 249-268.

Cliff, N., Keats, J. (2003) Ordinal measurement in the behavioural sciences, New Jersey: Lawrence Erlbaum.

Sutradhar, B.C. (2014). Longitudinal categorical data analysis, New York: Springer Science + Business Media

Szekli, R. (1995). Stochastic ordering and dependence in applied probability, Lecture Notes in Statistics 97, New York: Springer-Verlag

Stefănescu, M.L., Stefănescu, S. (2015). An indicator for measuring the evolution aspects for ordinal data, Paper presented at the 3rd International Conference ESPERA-2015, Bucharest, Romania, December 3-4, 2015, 10 pages.

Zheng, B. (2007). Inequality orderings and unit consistency, Social Choice and Welfare, vol. 29, no. 3, 515-538

***** (1990-2010). Data bases DQLR-RIQL, "Diagnosis for quality of life in Romania", Research Institute for Quality of Life, Romanian Academy 